3 Beeton CA, Bord S, Ireland D, et al. Osteoclast formation and bone resorption are inhibited by megakaryocytes. Bone 2006;39:985-90.

4 Mendes F, Farinha CM, Roxo-Rosa M, et al. Antibodies for CFTR studies. J Cyst Fibros 2004;3:69-72.

5 Henriksen K, Gram J, Schaller S, et al. Characterization of osteoclasts from patients harbouring a $\mathrm{G} 215 \mathrm{R}$ mutation in $\mathrm{ClC}-7$ causing autosomal dominant osteopetrosis type II. Am J Pathol 2004; 164:1537-45.

\section{Obstructive sleep apnoea in patients with type 2 diabetes}

An increased body mass index (BMI) is a risk factor for type 2 diabetes mellitus and obstructive sleep apnoea (OSA). The question whether this is just a shared risk factor or whether there is a deeper relationship has been addressed by West et $a l^{1}$ who used an initial screening Berlin questionnaire followed by overnight oximetry in selected respondents. OSA was found to be highly prevalent in this patient group. Although BMI was the best predictor of OSA, type 2 diabetes conferred a significant extra increase in the likelihood of having OSA after allowing for BMI, age and neck size.

We have examined the risk of OSA in a district general hospital diabetes clinic. We used the Berlin questionnaire and assessed sleepiness using the Epworth score in 63 people $(30$ women) with type 2 diabetes and a BMI of $>30 \mathrm{~kg} / \mathrm{m}^{2}$. Diabetic control was assessed using HbAlC. Thirty-five patients (56\%, 16 women) were found to have a high risk of OSA.

Despite the suggestion that improvement in sleep-disordered breathing using continuous positive airway pressure improves glucose intolerance in both the short and long term, no significant association was found between poor glycaemic control and the Berlin questionnaire risk group category. ${ }^{23}$

These results are similar to those of West et al and suggest the potential of a high burden of unrecognised OSA in people with diabetes. Furthermore, our findings are not restricted to the male population. We feel that clinicians who manage patients with type 2 diabetes should have a heightened awareness of the increased likelihood of OSA in this group. The Berlin questionnaire is easy to use and is an attractive alternative in the initial screening for OSA, particularly where access to sleep studies and oximetry is very limited.

Emer Kelly, Garret Cullen, Colm McGurk St Luke's Hospital, Kilkenny, Ireland

Correspondence to: Dr Emer Kelly, Department of Medicine RCSI, Smurfit Building, Beaumont Hospital, Dublin 9, Ireland; emerkelly@rcsi.ie

doi: $10.1136 /$ thx. 2006.074955

Competing interests/funding: None.

\section{References}

1 West SD, Nicoll DJ, Stradling JR. Prevalence of obstructive sleep apnoea in men with type 2 diabetes. Thorax 2006;61:945-50.

2 Harsch IA, Schahin SP, Radespiel-Troger M, et al. Continuous positive airway pressure treatment rapidly improves insulin sensitivity in patients with obstructive sleep apnea syndrome. Am J Respir Crit Care Med 2004; 169:156-62.

3 Brooks B, Cistulli PA, Borkman M, et al. Obstructive sleep apnea in obese noninsulin-dependent diabetic patients: effect of continuous positive airway pressure treatment on insulin responsiveness. J Clin Endocrinol Metab 1994;79:1681-5.

\section{Screening for type 2 diabetes in patients with obstructive sleep apnoea}

We read with much interest the findings in the recent paper by West et $a l^{1}$ of the prevalence of obstructive sleep apnoea (OSA) in men with type 2 diabetes. Although there may have been selection bias in the questionnaire respondents, the findings support the hypothesis that OSA is common in this population and is likely to be underdiagnosed. OSA is known to be independently associated with an increase in the cardiovascular risk factors that comprise the metabolic syndrome, ${ }^{2}$ including diabetes mellitus and impaired glucose tolerance. ${ }^{3}$

We have reviewed our data on 156 successive patients with OSA recently diagnosed by polysolmnography, 114 of whom (72 men) had glucose measurements checked at the time of diagnosis. Sixteen patients (14\%) were already known to have diabetes or impaired glucose tolerance. Although only five newly diagnosed cases of diabetes were identified, a further two had a single raised fasting glucose level and four had raised non-fasting glucose levels. Thus, a total of 11 patients $(9.6 \%)$ were identified by the screening process as potentially having diabetes or impaired glucose tolerance.

Unsurprisingly, the patients with diabetes or impaired glucose tolerance had higher mean body mass indices ( 37 vs $33.2 \mathrm{~kg} / \mathrm{m}^{2}$ ), but there seemed to be little difference in either the Epworth score (11.8 vs 9.9) or in the apnoeahypopnoea index (22.6 vs 24.4). These data support active screening of patients with newly diagnosed OSA for diabetes in order to allow earlier recognition and treatment.

C Snelson, R Chandrappa, H Moudgil

Department of Respiratory Medicine, Shrewsbury and Telford Hospitals NHS Trust, Apley Castle, Telford, UK

Correspondence to: Dr H Moudgil, Princess Royal Hospital, Apley Castle, Telford TF1 6TF, UK; h.moudgil@thedoctor.co.uk

Competing interests: None.

\section{References}

1 West S, Nicoll D, Stradling J. Prevalence of obstructive sleep apnoea in men with type 2 diabetes. Thorax 2006;61:945-50.

2 Coughlin S, Mawdsley L, Mugarza J, et al. Obstructive sleep apnoea is independently associated with an increased prevalence of metabolic syndrome. Eur Heart J 2004; 25:735-41.

3 Meslier N, Gagnadoux F, Giraud P, et al. Impaired glucose-insulin metabolism in males with obstructive sleep apnoea syndrome. Eur Respir J 2003;22:156-60. 